Original article

\title{
A modeling study on the sustainability of a certification-and-monitoring program for paratuberculosis in cattle
}

\author{
Pauline EZANNO ${ }^{\mathrm{a} *}$, Gerdien VAN SCHAIK ${ }^{\mathrm{b}}$, Maarten F. WEBER ${ }^{\mathrm{b}}$, \\ Johan A.P. HEESTERBEEK ${ }^{\mathrm{c}}$ \\ a Veterinary School \& INRA, Unit of Animal Health Management, BP 40706, \\ 44307 Nantes Cedex 03, France \\ b Animal Health Service, PO Box 9, 7400 AA Deventer, The Netherlands \\ ${ }^{\mathrm{c}}$ Faculty of Veterinary Medicine, Unit of Farm Animal Health, Utrecht University, \\ Yalelaan 7, 3584 CL Utrecht, The Netherlands
}

(Received 29 September 2004; accepted 5 April 2005)

\begin{abstract}
Certification-and-monitoring programs for paratuberculosis are based on repetitive herd testing to establish a herd's health status. The available tests have poor sensitivity. Infected but undetected herds may remain among certified "paratuberculosis-free" herds. The objective was to determine if truly free herds acquire a certified status and keep it over time when infected but undetected herds remain. The Dutch program was used as a basis to construct a mechanistic deterministic model of the evolution over 25 years of the number of herds per health status. Three health states for herds were defined: not detected as infected in the certification process to obtain a free status; not detected as infected by any of the repetitive tests for monitoring the certified free status; detected as infected. Among undetected herds, two types were defined: truly free versus undetected but infected. Transitions between states were due to the purchase of an infected animal, infection via the environment, clearance via culling or sales, detection of an infected animal, and certification. A sensitivity analysis was carried out. We showed that - for a $100 \%$ specific test only - most of the truly free herds at the beginning of the program got a certified free status and kept it over time. Most infected herds were either detected as infected or cleared. The number of certified truly free herds increased with a decrease in the animal-level prevalence or in the risk of purchasing an infected cattle, for example by restricting purchases to cattle from herds at the highest level of certification.
\end{abstract}

dairy cattle / paratuberculosis / certification / modeling

\section{INTRODUCTION}

Paratuberculosis, or Johne's disease, is an infection of economic importance for cattle farmers, because of decreasing milk production, premature culling, decreased fertility and decreased slaughter value of the carcass [5]. For instance, the decrease in milk production in sub-clinically infected dairy cows has been estimated to range between 5 and $25 \%[1,11,16]$. The etiologic agent of paratuberculosis is Mycobacterium avium subsp. paratuberculosis (MAP). The zoonotic implications of paratuberculosis are still

* Corresponding author: ezanno@vet-nantes.fr 
unclear but it makes participation in control programs more urgent in case paratuberculosis is proven to be a zoonosis.

Herds known to be at low risk to be infected are important as a source of uninfected cattle for trade. However, the identification of herds at low risk to be infected is difficult for two main reasons. First, diagnostic tests to detect paratuberculosis-infected cattle have a poor negative predictive value due to a low sensitivity of tests [20]. Cattle remain sub-clinical for a long time after infection. Excretion of MAP in the feces from infected cattle may begin before the development of clinical signs and before an antibody response. Therefore, new infections may occur before the first infected animal has been identified and culled. Second, herds at low risk to be infected have a low withinherd prevalence of infection, resulting in a low herd sensitivity and therefore in a low probability of detection [4]. Even when all animals test negative, the herd cannot be guaranteed free from paratuberculosis.

Certification-and-monitoring programs to determine and certify the health status of herds have been implemented in several countries. In countries such as Sweden, where the prevalence of infected dairy herds is very low $(<0.1 \%$; [2]), the objective is to maintain a favorable status and to eradicate the pathogen [17]. In other countries, such as in The Netherlands, the USA and Australia, control programs have been implemented for several years in order to limit the spread of the disease. In these countries, programs are formalized to classify herds based on the likelihood of being MAP-infected [5]. Such programs help to limit the spread of paratuberculosis by creating a disease-free source of replacement for cattle buyers. Several health states of herds are defined, based on successive herd tests, given a low rate of introduction of the infection into herds. The likelihood of becoming MAP-free increases with the number of negative results to herd tests. Programs can differ through the testing techniques (serology or fecal culture), the time interval between successive herd tests, the selection of animals in the herds to be tested, imposed rules for the purchase of animals and through actions taken after positive test results.

In most certification-and-monitoring programs, all or a sample of the animals in each herd are tested by either serology (ELISA; generally $\geq 3$ years of age) or fecal culture (individual or pooled; $\geq 2$ years of age). Both tests have a poor sensitivity, resulting in a large number of undetected but infected animals (false negative). Hence, undetected herds can persist in the certified group of herds. These herds may sell infected animals to truly MAP-free herds in the same or lower level of certification. It is questionable whether truly MAP-free herds buying cattle will keep their certification over time in the presence of undetected but infected herds in the same or higher level of the certification-and-monitoring program, even if the proportion of infected animals in these herds is low. The purchase of MAP-infected animals is thought to be the main cause of the introduction of the pathogen in a MAPfree herd [18]. In order to reduce the risk of introducing an infection in a MAP-free herd, purchasing animals from herds at a lower level of certification (lower number of negative annual examinations of the herd) is generally not allowed. However, contamination of cattle herds can also arise through the environment $[6,7]$. The pathogen can survive outside a host for around one year [12]. Moreover, MAP infects other species, such as sheep, birds, rabbits and other rodents, and is present in their excreta $[6,7]$, which can lead to a contamination of farm stored feed and pastures. Of course, repeating herd tests at regular intervals increases the probability of discovering infected cattle. However, the number of years required for true certification needs to be balanced against the financial feasibility of the scheme.

In the present paper, the objective was to test whether the certified MAP-free group of herds was sustainable, with either an 
increasing or a stable number of certified herds, or if there was a loss in certified herds. The Dutch paratuberculosis certification-and-monitoring program was used as a basis to construct a mechanistic model for the evolution of the health status of herds involved in the program. A sensitivity analysis was carried out to test if an alternative control strategy could improve the results and to determine the limits of the present program.

\section{MATERIALS AND METHODS}

\subsection{Mathematical model}

\subsubsection{Assumptions}

Young stock was assumed not to participate in the infection process (no shedders among the young stock).

The environmental force of infection represents the probability to be infected via the environment, because of for example pathogen survival as a free organism, propagation of the pathogen by air or by other indirect routes. It was assumed to be constant and not to depend on the within-herd prevalence or on the herd density.

Among undetected herds, the within-herd prevalence was assumed to vary. At the lowest level of the certification scheme, it was assumed to follow a beta distribution, which did not change over time (constant mode and percentiles). The within-herd prevalence at higher levels of certification and in detected herds was assumed to be based on the distribution of the prevalence at the lowest level and on the test characteristics.

Herds detected as infected were assumed to cull all the detected animals and to replace them by animals purchased in undetected herds of any level of the certification scheme. It was assumed that no additional test was performed on the remaining cattle or on samples of purchased animals.

Animals were assumed to be bought only from herds in the same or higher levels of certification. In each level of certification, all animals were assumed to have the same probability of being purchased.

Additional explicit assumptions on the mechanisms and relations of the model are given below in the model description and in the Methods Section 2.2.

\subsubsection{State variables}

Two levels of certification were represented (1 and 2) with $d$ annual tests being performed to go from level 2 (the lowest level of certification) to level 1 (the highest level of certification). For each level, two types of herds were considered: undetected but infected herds $(U)$ and truly MAP-free herds $(S)$. Detected herds were specifically identified (group I). In total, the five state variables included in the model were the number of $S_{2}, U_{2}, S_{1}, U_{1}$ and $I$ herds. Moreover, for groups $U_{2}, U_{1}$ and $I$, the corresponding variables were allowed to depend on the prevalence of MAP infection, denoted by $p$.

\subsubsection{Model description}

The total number of herds in the scheme (N) remained constant: $N=S_{1}+U_{1}+S_{2}+$ $U_{2}+I$. The transitions between states and their rates are depicted in Figure 1. The model is given by the following system of differential equations, with parameters and variables defined in Tables I and II, respectively:

$$
\begin{aligned}
\frac{d S_{1}}{d t}= & -\left[e+\left(1-l_{1}\right)\left(1-m_{1}\right)\right] S_{1}-(1-H S P) S_{1} \\
& +\int_{0}^{1} c(p) U_{1}(p) d p+\frac{H S P}{d} S_{2} \\
\frac{d U_{1}}{d t}= & {\left[e+\left(1-l_{1}\right)\left(1-m_{1}\right)\right] S_{1} } \\
& -\int_{0}^{1} c(p) U_{1}(p) d p-\int_{0}^{1} H S E(p) U_{1}(p) d p \\
& +\int_{0}^{1}[1-H S E(p)]^{d-1} U_{2}(p) d p
\end{aligned}
$$




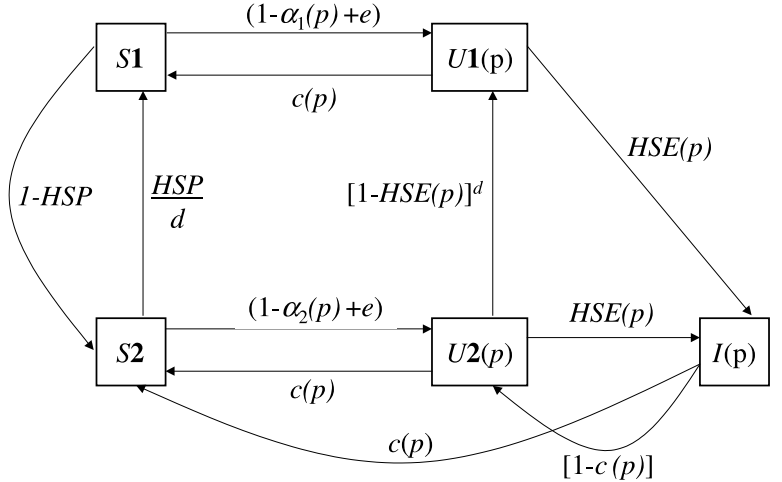

Figure 1. States and transitions between states.

$$
\begin{aligned}
\frac{d S_{2}}{d t}= & (1-H S P) S_{1}-\frac{H S P}{d} S_{2} \\
& -\left[e+\left(1-l_{2}\right)\left(1-m_{2}\right)\right] S_{2} \\
& +\int_{0}^{1} c(p) U_{2}(p) d p+\int_{0}^{1} c(p) I(p) d p \\
\frac{d U_{2}}{d t} & =\left[e+\left(1-l_{2}\right)\left(1-m_{2}\right)\right] S_{2} \\
& -\int_{0}^{1} c(p) U_{2}(p) d p-\int_{0}^{1} H S E(p) U_{2}(p) d p \\
& -\int_{0}^{1}[1-H S E(p)]^{d-1} U_{2}(p) d p \\
& +\int_{0}^{1}[1-c(p)] I(p) d p
\end{aligned}
$$

$$
\begin{aligned}
\frac{d I}{d t} & =\int_{0}^{1} \operatorname{HSE}(p) U_{1}(p) d p \\
& +\int_{0}^{1} H S E(p) U_{2}(p) d p-I .
\end{aligned}
$$

The status of a herd can change in several ways. A truly MAP-free herd $\left(S_{1}\right.$ or $\left.S_{2}\right)$ can buy an infected animal if open (with a prob- ability $\left\{1-m_{\mathrm{k}}\right\}, k \in\{1,2\}$ respectively), or becomes contaminated via the environment (with a probability $e$ ). It then becomes an undetected herd ( $U_{1}$ or $U_{2}$, respectively). A truly MAP-free herd can be detected as infected if the test specificity is not perfect (false positive, with probability $\{1-H S P\}$ ) and then goes to the group $S_{2}$ because it is still truly MAP-free. After $d$ successive negative herd tests, $U_{2}$ and $S_{2}$ herds become certified and go to groups $U_{1}$ and $S_{1}$, respectively. Because the test is assumed to be almost $100 \%$ specific (or $S P$ is very close to 1 in sensitivity analyses), the yearly transition rate from group $S_{2}$ to group $S_{1}$ can be approximated by $H S P / d$. Because the test sensitivity is not close to 1 , the transition rate from group $U_{2}$ to group $U_{1}$ cannot be simplified: an infected but undetected herd is certified after $d$ successive false negative tests with a probability $(1-H S E)^{d}$. An infected herd $\left(U_{1}, U_{2}\right.$ and $\left.I\right)$ can be cleared by chance because of the sale or culling of all its infected animals (with probability $c$ ). It then becomes a truly MAP-free herd $\left(S_{1}\right.$ or $\left.S_{2}\right)$. An infected but undetected herd $\left(U_{1}\right.$ and $U_{2}$ ) can be detected as being infected (with a probability $H S E$ ). It then becomes an $I$ herd. HSE and $c$ depends on the withinherd prevalence $p$. Detected herds do not stay in group $I$ but become either truly free $\left(S_{2}\right.$; with probability $\left.c\right)$ or undetected but infected $\left(U_{2}\right.$; with probability $\left.1-c\right)$ herds. 
Table I. Parameter initial values and ranges of values for sensitivity analyses, based on expert opinions and on the literature, for Dutch dairy herds.

\begin{tabular}{|c|c|c|c|c|}
\hline Parameter & Value & Range of values & Definition & Ref. \\
\hline$a_{1}$ & 0.02 & $0.00-0.20$ & Yearly purchasing rate ${ }^{\mathrm{a}}$ in herds of group 1 & * \\
\hline$a_{2}$ & 0.04 & $0.00-0.20$ & Yearly purchasing rate ${ }^{\mathrm{a}}$ in herds of group 2 & $*$ \\
\hline$d$ & 4 & $1-10$ & Duration of the certification process (in years) & [19] \\
\hline$e$ & 0.001 & $0.00001-0.05$ & Probability of contamination through the environment & $\begin{array}{c}{[6,7,12]} \\
*\end{array}$ \\
\hline$l_{1}$ & 0.90 & $0.00-1.00$ & Proportion of closed herds in group 1 & $*$ \\
\hline$l_{2}$ & 0.55 & $0.00-1.00$ & Proportion of closed herds in group 2 & * \\
\hline$S_{2}$ ini & 0.20 & $0.00-1.00$ & Initial proportion of truly MAP-free herds in group 2 & [10] \\
\hline$\mu$ & 0.30 & $0.10-0.40$ & Yearly renewal rate in all herds & [10] \\
\hline$p_{\text {mode }}$ & 0.05 & $0.01-0.25$ & $\begin{array}{l}\text { Mode of the beta distribution of the within-herd } \\
\text { prevalence in } U_{2} \text { herds }\end{array}$ & * \\
\hline$p_{95}$ & 0.25 & $0.05-0.45$ & $\begin{array}{l}\text { 95th percentile of the beta distribution of the within- } \\
\text { herd prevalence in } U_{2} \text { herds }\end{array}$ & $*$ \\
\hline$S E$ & 0.33 & $0.20-0.70$ & Test sensitivity & {$[13,20]$} \\
\hline$S P$ & 1.000 & $0.996-1.000$ & Test specificity & {$[13,20]$} \\
\hline$s$ & 1 & $0.25-1.00$ & Sampling proportion & \\
\hline$T$ & 65 & $50-100$ & Herd size & {$[10,19] *$} \\
\hline
\end{tabular}

* Expert's opinions.

a The purchasing rate is the number of cattle purchased per year per animal present in the herd.

Table II. Definition of the variables used in the model.

\begin{tabular}{lc}
\hline Variable & Definition \\
\hline$m_{\mathrm{k}}$ & $\begin{array}{c}\text { Yearly probability for a herd in } \\
\text { group } k \text { of purchasing only } \\
\text { uninfected animals }\end{array}$ \\
$c(p)$ & $\begin{array}{c}\text { Yearly probability of clearance for a } \\
\text { herd with within-herd prevalence } p\end{array}$ \\
$H S E(p)$ & $\begin{array}{c}\text { Herd sensitivity of the test for a herd } \\
\text { with within-herd prevalence } p \\
\text { HSP }\end{array}$ \\
\hline
\end{tabular}

\subsection{Methods}

\subsubsection{Initial conditions and output variables}

Because of the high number of states in the model, the analytic behavior of the model could not be explored and numerical simulations were performed in Excel for Windows. The implemented model was discrete with a time step of one year. It represented changes over time in numbers of herds per state variable. As an illustrative example we took the Dutch paratuberculosis certification-and-monitoring program. We modeled a simplified version of this scheme, including only two levels of the certification-and-monitoring program. Before entering the lowest level of the Dutch program, a first herd test consists of a serial testing of all cattle $\geq 3$ years of age by serology (ELISA) and individual fecal culture of seropositive animals. In our model, this initial step was not modeled but we took into account the assumed results of the first herd examination to define an initial classification of herds into groups $U_{2}$ and $S_{2}$. We started our simulations with herds that were test-negative to ELISA or test-positive to ELISA and test-negative to individual fecal 
culture. The second to fifth annual herd tests consist of serial testing of all cattle $\geq 2$ years of age with pooled fecal culture and individual-animal fecal culture of positive pools [19]. In our model, group $S_{1}$ contained truly MAP-free herds at the highest level of certification. Group $U_{1}$ contained infected but undetected herds at the highest level of certification. Group $S_{2}$ contained truly MAP-free herds with one negative serological herd test and 0 to 3 negative herd tests by pooled fecal cultures. Group $U_{2}$ contained infected but undetected herds with one negative serological test and 0 to 3 negative herd tests by pooled fecal cultures. In groups $S_{2}$ and $U_{2}$, the herds were assumed to be evenly distributed over the statuses in the program (intermediate levels of certification). Group I contained herds that were infected and test-positive at the last herd test by pooled fecal culture.

At the start of the simulation, all the herds were in group $2\left(S_{2}\right.$ and $U_{2}$, i.e. lower level of certification). In a field study in Dutch dairy herds [14], $61 \%$ of 90 herds were testpositive. As a worst-case scenario, $20 \%$ of the herds were assumed here to be initially truly MAP-free (group $S_{2}$ ). In this study, only the 1000 original herds were considered and therefore the total number of herds stayed constant over time. The simulations were performed on a 25-year horizon with a yearly time step. The output variables were the following:

- the number of herds in each group over time,

- the animal-level prevalence over time,

- the number of herds changing status per time step and per status.

\subsubsection{Parameterization}

The characteristics of Dutch dairy herds and of the Dutch paratuberculosis certification-and-monitoring program were used to parameterize the model ([10, 19]; expert's opinions; Tab. I). The mean herd size $(T)$ was 65 cattle older than two years of age, which corresponds to the tested animals. In herds increasing their level of certification, it has been observed that farmers tend to decrease the risk of introducing infected cattle. Observations on Dutch dairy herds showed that the percentage of closed herds was much higher in herds participating in the certification-and-monitoring program than in non-participating herds, whereas the overall purchasing rate was lower. Parameters of our model were chosen according to these observations. Among $S_{2}$ and $U_{2}$ dairy herds, $55 \%$ of the herds were closed. The yearly purchasing rate in open $S_{2}$ and $U_{2}$ herds was $4 \%$. Among $S_{1}$ and $U_{1}$ dairy herds, $90 \%$ of the herds were closed. The yearly purchasing rate in open $S_{1}$ and $U_{1}$ herds was $2 \%$. In all herds, the renewal rate was $30 \%$.

All the animals were tested $(s=1)$ through pooled fecal culture, which was assumed to have a perfect specificity $(S P=1)$ and a sensitivity $(S E)$ of $0.33[13,20]$. The duration of the scheme $(d)$ was four years. Contamination through the environment was assumed to arise at a yearly rate of 0.001 herd $^{-1} \cdot \mathrm{yr}^{-1}$. The environmental force of infection was assumed to be independent of the herd density or of the herd-level and animal-level prevalences, because no information was available to quantify such parameters.

Based on experts' opinions, the withinherd prevalence in $U_{2}$ herds was assumed to follow a beta distribution with mode $5 \%$, mean $11 \%$ and 95 th percentile $25 \%$ (Fig. 2). Whatever the number of herds in group $U_{2}$, the distribution did not change over time. A proportion of herds in group $U_{2}$ had a within-herd prevalence $p$. The evolution of the status of these herds depended on the herd test sensitivity (HSE) and the probability of clearance $(c)$, which varied with the within-herd prevalence $p$ (see below). The distributions of the within-herd prevalences in group $U_{1}$ and $I$ were evaluated based on the distribution in group $U_{2}$.

In $S_{\mathrm{k}}$ herds $(k \in\{1,2\})$, no infected animals can be purchased in closed herds (in proportion $l_{\mathrm{k}}$ ). In open herds (in proportion $\left.\left\{1-l_{\mathrm{k}}\right\}\right)$, the probability that none of the $a_{\mathrm{k}} T$ 


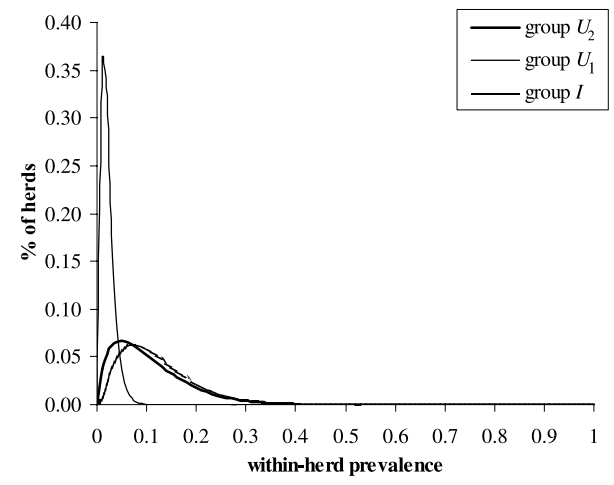

Figure 2. Distributions of the within-herd prevalences in groups $U_{2}$ (Beta $(1.71 ; 14.49)$; bold black), $U_{1}$ (calculated; black) and $I$ (calculated; grey).

purchased animals was infected was $m_{\mathrm{k}}$. The herds were assumed to purchase animals only from herds in the same or higher level of certification. Hence, for open herds in group $S_{1}$ ( $S_{2}$ respectively), the purchased animals were disease free if they came either from $S_{1}$ herds ( $S_{1}$ or $S_{2}$ herds respectively) or from the uninfected proportion $(1-p)$ of the animals in undetected herds of prevalence $p$ : $U_{1}(p)\left(U_{1}(p)\right.$ or $U_{2}(p)$ respectively). The probabilities to purchase only MAP-free animals were respectively:

$$
\begin{aligned}
& m_{1}=\left[1-\frac{\int_{0}^{1} p U_{1}(p) d p}{S_{1}+U_{1}}\right]^{a_{1} T} \text { and } \\
& m_{2}=\left[1-\frac{\int_{0}^{1} p\left[U_{1}(p)+U_{2}(p)\right] d p}{S_{1}+U_{1}+S_{2}+U_{2}}\right]^{a_{2} T} .
\end{aligned}
$$

The probability of clearance $c(p)$ of an undetected but infected herd with prevalence $p$ depended on the culling or the sale of infected animals, related to the renewal rate $\mu$. If $\mu<p$, no clearance occurred. Otherwise, $c$ was the probability to have exactly $p T$ infected animals among the $\mu T$ animals that left the herd. Culled animals were assumed to be randomly selected in the herd. Infected animals were assumed to be randomly distributed through the herd. If $p T$ and $\mu T$ were integers, the probability of clearance would follow a hypergeometric distribution. Since they had decimal values, the gamma function was used to calculate $c(p)$ as follows:

$c(p)=\left\{\begin{array}{cc}0 & \text { if } \mu<p \\ \frac{\Gamma(\mu T) \Gamma(T-p T)}{\Gamma(\mu T-p T) \Gamma(T)} & \text { else }\end{array}\right.$.

The probability of clearance depended on $p$, which varied in a group $\left(U_{1}, U_{2}\right.$ and $\left.I\right)$. Hence, the number $n_{k}$ of herds that were cleared in a time step in a group $k$ was the sum of the number of herds in that group that were cleared when having a given prevalence $p$ :

$$
n_{k}=\int_{0}^{1} c_{k}(p) U_{k}(p) d p .
$$

The herd sensitivity depended on the within-herd prevalence $(p)$ :

$\operatorname{HSE}(p)=1-[1-S E p-(1-S P)(1-p)]^{T}$.

The herd specificity $(H S P)$ was $H S P=$ $S P^{T}$, with value 1.00 for a $100 \%$ specific test.

\subsubsection{Sensitivity analyses}

The model is deterministic and describes therefore only one potential dynamic outcome of the system, given the assumptions, parameter choices and initial conditions. In order to take into account the influence of changing the parameter values on the results, a sensitivity analysis was performed. We evaluated the variation in the number of $S_{1}$ herds after 25 years to a change in the parameters (Tab. I). First, we analyzed the model sensitivity to a change in the initial proportion of truly MAP-free herds $\left(S_{2}\right.$ herds). Then, we analyzed the model sensitivity to a change in herd characteristics: renewal rate $(\mu)$, purchasing rate in groups 1 
or $2\left(a_{1}, a_{2}\right)$, proportion of closed herds in groups 1 or $2\left(l_{1}, l_{2}\right)$, and herd size $(T)$. By default, herd testing included all adult animals ( $\geq 2$ years) in a herd. Alternatively, only a proportion of adult animals was randomly sampled. We analyzed the model sensitivity to a change in the proportion of tested animals $(s)$. Next, we analyzed the model sensitivity to a change in disease characteristics: mode of the within-herd prevalence distribution $\left(p_{\text {mod }}\right)$ and its 95 th percentile $\left(\mathrm{p}_{95}\right)$ in group $U_{2}$ and environmental force of infection $(e)$. Finally, we analyzed the model sensitivity to a change in the certification scheme characteristics: sensitivity $(S E)$ and specificity $(S P)$ of the pooled fecal culture and duration of the certification program $(d)$.

\subsubsection{Evaluation of an alternative control strategy}

An alternative strategy to control the risk of introduction of an infected animal is to restrict the purchases for $U_{2}$ and $S_{2}$ herds to animals from certified herds only ( $S_{1}$ and $U_{1}$, since they cannot be distinguished in reality). This strategy was evaluated with the model according to the number of truly MAP-free herds $\left(S_{1}\right)$ after 25 years and the evolution in the global animal-level prevalence over time.

\section{RESULTS}

\subsection{Distributions of the within-herd prevalence in groups $U_{1}$ and $I$}

The distributions of the within-herd prevalence in groups $U_{1}$ and $I$ calculated at each step of the simulation did not change over time. In group $U_{1}$, the mode of the distribution of the within-herd prevalence was $1 \%$, with $36 \%$ of the herds having a lower prevalence. In group $I$, the mode of the distribution was $8 \%$, with $36 \%$ of the herds having a lower prevalence (Fig. 2). More than $97 \%$ of the herds in group $U_{1}$ had a prevalence
Table III. Averaged yearly transition rates (\%).

\begin{tabular}{lccccc}
\hline $\begin{array}{l}\text { To } \\
\text { From }\end{array}$ & $S_{1}$ & $U_{1}$ & $S_{2}$ & $U_{2}$ & $I$ \\
\hline $\mathrm{S}_{1}$ & 99.9 & 0.1 & & & \\
$U_{1}$ & 25.1 & 45.7 & & & 29.2 \\
$S_{2}$ & 23.5 & & 70.5 & 6.0 & \\
$U_{2}$ & & 2.4 & 3.8 & 16.9 & 76.9 \\
$I$ & & & 1.5 & 98.5 & \\
\hline
\end{tabular}

lower than $5 \%$. Less than $5 \%$ of the herds in group I had a prevalence higher than $26 \%$.

\subsection{Results for the certification process and monitoring}

Per year, only 0.4 (i.e. 8.8 in the 25 years of the simulation) certified and truly MAPfree herds $\left(S_{1}\right)$ became infected but undetected herds $\left(U_{1}\right)$, representing $0.1 \%$ of the yearly transition rates from $S_{1}$ status (Tab. III). This demonstrates the sustainability of the certification status. Because the test specificity was one, none of the $S_{1}$ herds became uncertified truly MAP-free herds $\left(S_{2}\right)$. More $S_{2}$ herds became certified $\left(S_{1} ; 18\right.$ herds per year on average $)$ than infected $\left(U_{2} ; 5\right.$ herds per year on average). Almost as many $U_{1}$ herds were cleared as detected ( $S_{1}$ vs. $I$; 4 herds per year on average). Most of the $U_{2}$ herds were detected as infected $(I ; 260$ herds per year on average); few became either certified $\left(U_{1} ; 8\right.$ herds per year on average) or cleared $\left(S_{2} ; 13\right.$ herds per year on average). Very few detected herds $(I)$ were cleared after detection $\left(S_{2} ; 4\right.$ herds per year on average).

The number of $S_{1}$ herds rapidly increased in the simulation (Fig. 3), with an increase of more than 15 herds per year. Thus, with the present set of parameters, all herds will slowly become certified. The $U_{1}$ and $S_{2}$ herds were transition states from uncertified herds $\left(U_{2}\right.$ and $\left.I\right)$ to certified and truly MAP-free herds $\left(S_{1}\right)$.

The global animal-level prevalence of infection in the population of herds in which animals can be purchased (all except $I$ herds) decreased with time (Fig. 4). 


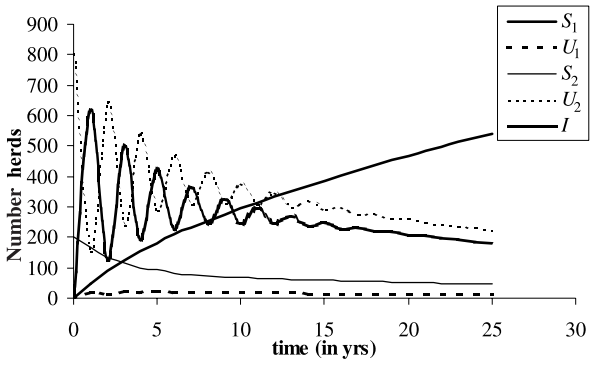

Figure 3. Dynamics of the number of herds per status $\left(S_{1}\right.$ in bold black, $U_{1}$ in dotted bold black, $S_{2}$ in black, $U_{2}$ in dotted black, $I$ in hatched grey) during 25 years.

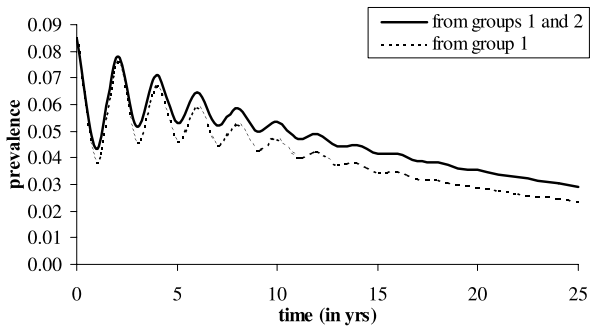

Figure 4. Variation over time of the animallevel prevalence in all but $I$ herds, with herds in group 2 purchasing cattle from herds in both groups 1 and 2 (bold black), or from herds in group 1 only (hatched black).

\subsection{Sensitivity analyses}

\subsubsection{Basic model}

The number of $S_{1}$ herds after 25 years increased with an increasing initial number of truly MAP-free herds ( $S_{2}$ herds) in the population, i.e. with a decreasing initial herdlevel prevalence (Fig. 5). When no herds were initially truly MAP-free (all herds were initially infected but undetected, the herdlevel prevalence being one), the number of $S_{1}$ herds after 25 years was still above $40 \%$ of the total number of herds.

\subsubsection{Herd characteristics}

The larger the herds were, the lower was the number of $S_{1}$ herds after 25 years (Fig. 6). The number of $S_{1}$ herds after 25 years also

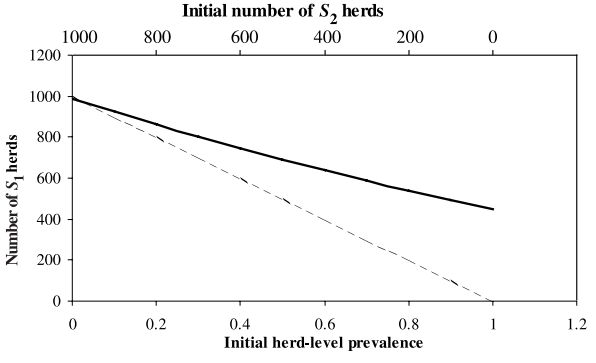

Figure 5. Number of $S_{1}$ herds after 25 years according to the initial proportion of truly MAPfree herds $\left(S_{2}\right.$ herds), i.e. the initial herd-level prevalence.

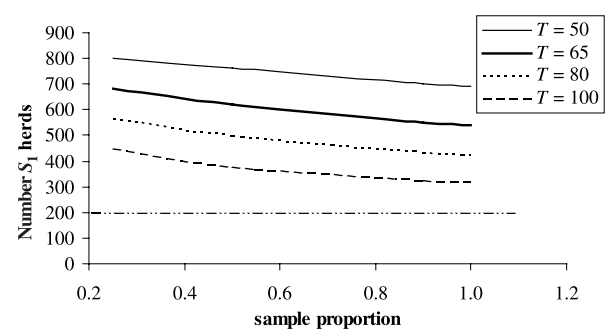

Figure 6. Number of $S_{1}$ herds after 25 years according to the herd size $(T)$ and the sampling proportion. Other parameters were kept at their initial value.

decreased with an increasing proportion of tested animals in the herd (Fig. 6).

A change in the renewal rate $(\mu)$ hardly had any effect on the number of $S_{1}$ herds after 25 years (results not shown).

The effects of the purchasing rate $(a)$ and of the proportion of closed herds $(l)$ on the number of $S_{1}$ herds after 25 years were closely related (Fig. 7). The number of $S_{1}$ herds after 25 years decreased if the purchasing rate in any group ( 1 or 2 ) increased and the proportion of closed herds decreased. A change in the parameters of group 2 had a larger effect on the response than a change in the parameters of group 1. A small increase in the purchasing rate of herds in the lowest level of certification $\left(a_{2}\right.$; Fig. 7a) resulted in an important decrease in $S_{1}$ herds, even if the proportion of closed herds $\left(l_{2}\right)$ was 

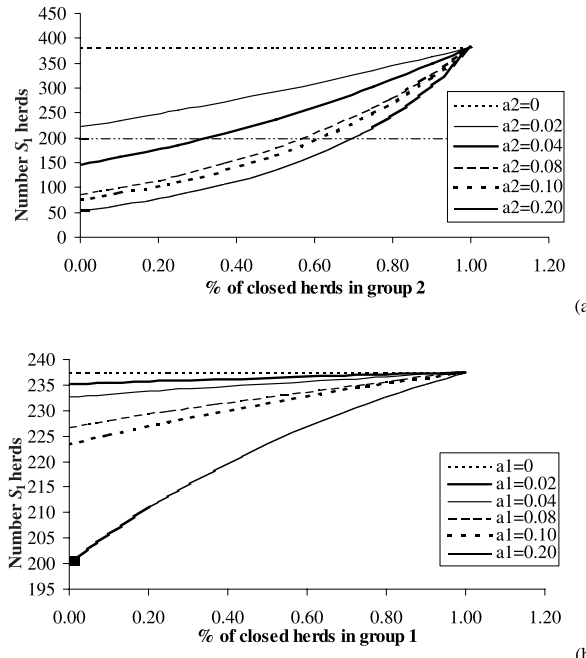

(b)

Figure 7. Number of $S_{1}$ herds after 25 years according to the purchasing rate $\left(a_{\mathrm{i}}\right.$ in group $\left.i\right)$ and the proportion of closed herds; (a) in group 2; (b) in group 1; y-scales are different. Other parameters were kept at their initial value.

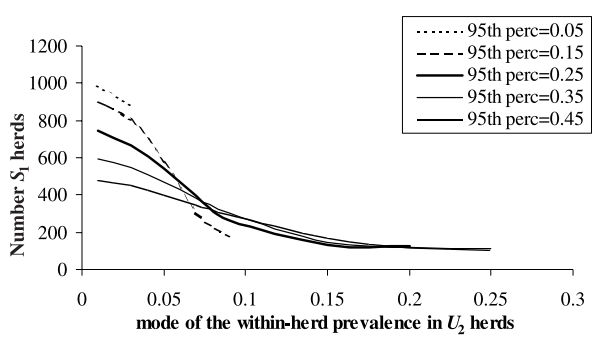

Figure 8. Number of $S_{1}$ herds after 25 years according to the mode of the distribution of the within-herd prevalence of infection in $U_{2}$ herds and to its 95th percentile. Other parameters were kept at their initial value.

high. On the contrary, even a large increase in $a_{1}$ resulted only in a small decrease in $S_{1}$ herds, while $l_{1}$ was above $70 \%$ (Fig. 7b). The effect became larger for lower $l_{1}$ values or for $a_{1}$ about $20 \%$.

\subsubsection{Disease characteristics}

The model was sensitive to the mode of distribution of the within-herd prevalence of infection in $U_{2}$ herds and its 95th percen-

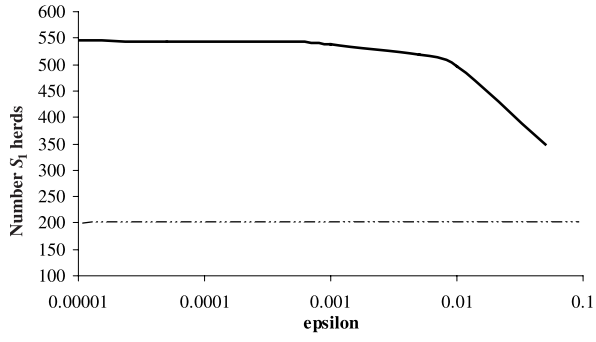

Figure 9. Number of $S_{1}$ herds after 25 years according to the rate of infection via the environment (logarithmic x-axis). Other parameters were kept at their initial value.

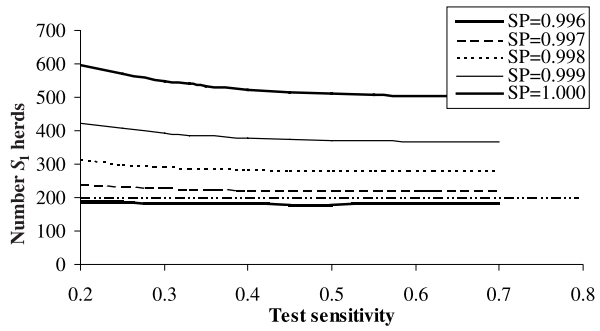

Figure 10. Number of $S_{1}$ herds after 25 years according to the test specificity $(S P)$ and sensitivity. Other parameters were kept at their initial value.

tile (Fig. 8). The lower the mode and the smaller its 95 th percentile, the higher the number of $S_{1}$ herds after 25 years. For a mode higher than $7 \%$, the decrease in the number of $S_{1}$ herds after 25 years decreased, even for large values of the 95 th percentile.

Until the environmental force of infection reached at least $0.005 \mathrm{herd}^{-1} \cdot \mathrm{yr}^{-1}$, the probability to be infected by the environment had no effect on the number of $S_{1}$ herds after 25 years (Fig. 9). An environmental contamination larger than 0.01 herd $^{-1} . \mathrm{yr}^{-1}$ resulted in a large decrease in the number of $S_{1}$ herds after 25 years, which could lead to zero $S_{1}$ herds after more than 25 years.

\subsubsection{Certification scheme}

The model was rather insensitive to the test sensitivity $(S E)$, even for lower withinherd prevalences of infection (results not 


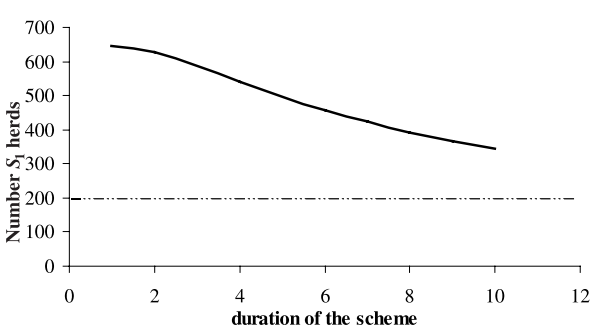

Figure 11. Number of $S_{1}$ herds after 25 years according to the duration of the certification process. Other parameters were kept at their initial value.

shown). On the contrary, a decrease in test specificity $(S P)$ had a large negative impact on the number of $S_{1}$ herds after 25 years (Fig. 10). With a specificity of 0.999 , the number of $S_{1}$ herds decreased rapidly and was halved in 25 years.

The quicker the certification process, the higher the number of $S_{1}$ herds after 25 years (Fig. 11). Less herd tests (4, 2 or 1 test) resulted in an increase in the mode and the 95th percentile of the distribution of the within-herd prevalence in the highest level of certification (mode of $1,2,3 \%$, respectively; 95th percentile of 5, 8, $12 \%$ respectively). As a consequence, there was a decrease in the yearly transition probability from $U_{1}$ to $S_{1}(25.1,15.2,9.0 \%$, respectively), but a very low increase in the yearly transition probability from $S_{1}$ to $U_{1}(0.11$, $0.14,0.19 \%$, respectively). However, there was an increase in the yearly number of herds that cleared in group 1 (from $U_{1}$ to $S_{1}$ : 3.6, 5.7, 6.5 herds per year, respectively). Furthermore, it resulted in an increase in the yearly transition probability from the lowest to the highest level of certification (from $U_{2}$ to $U_{1}: 2.4,7.7,19.2 \%$, respectively; from $S_{2}$ to $S_{1}: 23.5,47.1,93.9 \%$, respectively).

\subsection{Alternative control strategy}

The number of $S_{1}$ herds after 25 years was higher when herds in group 2 were allowed to purchase animals only from herds in group 1 compared to when they were allowed to purchase animals in both group 1 and group 2 (606 versus 540, respectively). Moreover, the global prevalence of infection at the animal level decreased more rapidly when purchases were restricted to group 1 than without such restriction (Fig. 4).

\section{DISCUSSION}

\subsection{Modeling approach}

Certification-and-monitoring programs are implemented to identify truly disease-free herds. However, a truly MAP-free status cannot be guaranteed because undetected but infected herds may remain among certified herds. The objective of our modeling study was to evaluate if truly MAP-free herds became certified and kept their status over time.

Our model represented the dynamics of the certification process and evaluated the effect of changing any parameters of the model on the number of truly MAP-free and certified herds after 25 years $\left(S_{1}\right)$. The dynamics showed oscillations, especially in the number of $U_{2}$ and $I$ herds. These oscillations were explained by the forced transitions between group $I$ and group $U_{2}$ : the herds in group $I$ were assumed to cull all the detected animals without performing any other test; in the next step they joined group $U_{2}$ or, less frequently, group $S_{2}$; then another test was performed and infected herds could be detected again. Simplifications were used and the within-herd disease spread was not modeled. However, it was possible with our model to test an alternative control strategy such as a restriction of purchases to animals from a specific group of herds. Moreover, key-parameters were identified that had a large impact on the sustainability of the certified status. To our knowledge, field data of long-term ( $\geq 20$ years) monitoring of MAP-unsuspected herds are not available. Thus, no direct validation was 
possible. The results obtained were coherent with the expert's opinions. As expected, the animal-level prevalence over the herds involved in the scheme decreased over time. Moreover, the distribution of the within-herd prevalence in $U_{1}$ herds had a $1 \%$ mode and a maximum value of $10 \%$, as expected and as also found by simulation in Weber et al. [19]. However, the model might be too optimistic in quantifying the number of $S_{1}$ herds after 25 years, since $40 \%$ of truly MAP-free herds seems hard to obtain if no herd was initially truly free in a true farming system with within-herd spread of MAP.

The present model was simple enough to be easily adaptable to other certification schemes and to be easy to handle. The model included the most critical points of a certification-and-monitoring scheme: characteristics of the test used for herd testing, herd and sampling characteristics, duration of the certification process before a diseasefree status is obtained. Some of the parameter values could not be obtained easily because no information was available in the literature. This included the proportion of infected animals in infected but undetected herds and the environmental force of infection. Therefore, a range of values was tested for "unknown" parameters to determine how sensitive the model was to those parameters.

Our model represented the dynamics of herd health status over time. Initially, all herds involved in the process have been test-negative to an ELISA or test-positive to an ELISA but test-negative to individual fecal cultures. The first herd examination of the process was not specifically modeled but was taken into account in the initial classification of herds: as a worst-case scenario, $20 \%$ of the herds were initially truly MAPfree $\left(S_{2}\right)$. The remaining $80 \%$ were undetected but infected $\left(U_{2}\right)$. In order to reflect the variability in within-herd prevalence among herds in group $U_{2}$, the proportion of infected animals per herd was chosen to be beta distributed. This distribution was chosen among other possible distributions since no further precise information was availa- ble. The distribution did not change over time, as for instance if the within-herd spread of MAP was modeled, and did not depend on the number of herds in a given status. The probability of clearance, the probability of detection of infected herds and the probability of infection via purchases depended on the within-herd prevalence. In reality, the proportion of infected animals in a herd may decrease because detected animals are culled, or may increase because of the within-herd infection process. In our model, the mode of the withinherd prevalence in $U_{1}$ herds was always $1 \%$, resulting in a high probability of clearance for most of the $U_{1}$ herds. However, this probability decreased rapidly for higher prevalences: it was 46, 21 and 9\% for prevalences of 1,2 and 3\%, respectively. Van Roermund et al. (2002, cited in [19]) included the evolution of the proportion of infected animals in a given infected herd in their model of the between-herd transmission of paratuberculosis. However, their objective was different since they aimed to quantify the between-herd transmission and to evaluate if an undetected but infected herd could give rise to the infection of, on average, more than one free-herd. Our objective was to evaluate if a certified truly MAP-free herd will keep its status over time and if the certified and truly MAP-free group of herds will be sustainable. The purchase of infected animals was the only route of infection of free herds in relation to undetected but infected herds. In our model, not only one, but several source-herds can sell infected animals to one free herd. Therefore, we focused on the whole group of undetected herds and the distribution of the within-herd prevalence in this group, instead of only one infected herd and the evolution of its prevalence over time. When a newly infected herd just enters group $U_{\mathrm{i}}$, the proportion of infected animals in this herd is likely to be lower than the mode of distribution of the within-herd prevalence in group $U_{\mathrm{i}}$. On the contrary, when a herd is detected as infected, its prevalence may be higher than the mode in its group $U_{\mathrm{i}}$. The 
infection process may also give rise to an increase of the within-herd prevalence in a given herd. Therefore, we assumed that overall the prevalence will remain constant and we chose to simplify the model and to analyze the effect of changes in the mode of the distribution on the model by sensitivity analyses.

Our model did not represent the young stock within herds. This simplification was made because we focused on shedding (infectious and detectable) animals. Young animals are known to be the most susceptible animals to paratuberculosis infection. However, infected young stock is hard to detect and serial herd tests generally focus on animals older than 24 months of age. Moreover, most infected young animals do not shed. The latency period can last several years, and animals shed at an older age. Consequently, representing young animals in the model may lead to an increase in undetected herds, a decrease in detection of infected herds, and a decrease in the clearing probabilities, i.e. a decrease in the transition from group $U$ to group $S$. However, it would have mainly influenced the time delay in identifying the truly MAP-free herds, which would not have dramatically changed the main results of our study.

\subsection{Certification and monitoring of paratuberculosis in cattle herds}

The simulated certification program led to sustainable results, with an increasing number of $S_{1}$ herds over time when the test was $100 \%$ specific. The test sensitivity did not influence the number of $S_{1}$ herds. On the contrary, the model of van Roermund et al. (2002, cited in [19]) was very sensitive to the test sensitivity, with decreasing betweenherd transmission for increasing test sensitivity. In our model, whatever the test sensitivity, a large proportion of undetected but infected herds were detected, even for low within-herd prevalences of infection. There was a balance between the detection of undetected but infected herds and their probability of clearance, when the test sensitivity was very low $(<20 \%)$, when the animals were not all tested, when the herds were certified after a lower number of tests than four tests or when the within-herd prevalence in the lowest level of certification was low. This was clearly demonstrated by the fact that more than $40 \%$ of the herds were certified and truly MAP-free after 25 years of simulation even when no herds were initially truly MAP-free. This can be explained by the fact that an increase in the herd-level prevalence did not change the distribution of the within-herd prevalence in $U_{2}$ herds, but only the number of herds with a given prevalence. Therefore, for a higher herd-level prevalence, there was an increase in the number of herds with low prevalence (that may be cleared during the certification process), other herds being generally detected as infected because of higher prevalence.

The increase in the number of $S_{1}$ herds after 25 years for shorter programs was the result of several changes in the process. As expected, fewer herd tests resulted in an increase in the number of certified herds (group 1). $S_{2}$ herds became rapidly certified. Once in group $S_{1}$, most of the herds were closed, open herds had a lower purchasing rate and purchased animals came only from group 1 . $U_{2}$ herds also became rapidly certified, resulting in an increase in the withinherd prevalence in the highest level of certification (group $U_{1}$ ). This increased the risk of purchasing infected animals. However, it only slightly influenced the transition probability from $S_{1}$ to $U_{1}$. This also increased the probability of detection and decreased the probability of clearance, i.e. decrease the transition probability from group $U_{1}$ to group $S_{1}$. However, since the number of $U_{1}$ herds was higher for shorter programs and since some herds still had low within-herd prevalences, the number of herds that were cleared (from $U_{1}$ to $S_{1}$ ) increased.

If the test was not $100 \%$ specific, the certification scheme was not sustainable anymore. This confirms the importance of 
using tests with the highest possible specificity, especially for herds at the highest level of certification ( $S_{1}$ and $U_{1}$ here), in order to prevent false positive results.

A large gain was obtained after a decrease in the proportion of infected animals in the early stages of the certification process (in group 2). This decrease in the number of infected animals in herds in group 2 corresponds to the situations where the efficiency of the transmission of MAP has been changed by herd management improvement.

An environmental force of infection was included in our model. It accounted for a contamination via a reservoir species, via survival of the pathogen in the environment, i.e. routes of transmission other than purchase of cattle. In the present study, the environmental infection pressure was not related to the prevalence of infection or to the density of herds. In reality, the environment is likely to be more polluted with increasing within-herd prevalence. For truly MAP-free and certified herds, i.e. that are likely to be truly MAP-free for several years, the environmental infection pressure may be lower than the average risk for all herds. However, since herds may be distributed in space independently from their certification level, neighboring herds may also influence the between-herd infection process. We simplified the model by neglecting the effect of the within-herd prevalence and of the local density of herds on the environmental force of infection. The model was insensitive to a change in the environmental force of infection for values lower than 0.05 herd $^{-1} \cdot \mathrm{yr}^{-1}$. This indicates that the risk for truly MAP-free herds to become infected via the environment remains negligible compared to the risk of infection via the introduction of infected animals when the environmental force of infection is truly less than 0.05 herd $^{-1} \cdot \mathrm{yr}^{-1}$.

Both the purchasing rate and the proportion of closed herds influenced the number of $S_{1}$ herds after 25 years. The purchasing rate is related to the risk of introduction of an infectious animal, whereas the propor- tion of closed herds is related to the number of herds at risk of being infected by purchases. The effect was smaller if the parameters of the herds in group 1 were changed than if the ones in group 2 were changed. The purchasing rate in certified herds had a strong effect on the between-herd transmission in the model of van Roermund et al. (2002; cited in [19]). However, they did not include in their model another route of infection, such as an environmental force of infection. In addition, they considered lower purchasing rates that were constant for all herds (no closed herds). Moreover, they did not consider several levels of certification with different prevalences and purchasing rates. In our model, when $90 \%$ of the herds in group 1 were closed (as observed in the field among herds that are certified), a change in the purchasing rate of group 1 only affected the probability of infection of $10 \%$ of the $S_{1}$ herds. Moreover, the withinherd prevalence in $U_{1}$ herds was low in our model, resulting in a low probability to be infected via purchasing animals from group 1 . As a result, the benefit to restrict purchases of animals from herds in group 1 for all the herds involved in the scheme has been shown. Furthermore, the subsequent advantage of certification-and-monitoring programs for producers involved and who have a certified herd will be higher animal selling prices.

\subsection{How to increase the number of certified herds?}

The sensitivity analyses have shown that a decrease in the within-herd prevalence in herds entering the certification process ( $p_{\text {mod }}$ in our model) will give rise to an increase in the number of certified herds. This is a result of three mechanisms: a decrease in the within-herd prevalence will give rise to an increase in the probability of clearance, to a decrease in the probability of detection of infected herds and to a decrease in the probability of infection of truly free herds. However, test-and-cull strategies 
alone do not sufficiently reduce the withinherd prevalence of paratuberculosis [5, 9]. Sensitivity of diagnostic tests is not high enough to detect all infected cattle. Therefore, if one of the objectives of a certificationand-monitoring program for paratuberculosis is to increase the number of certified herds, it should be combined with improved herd management. This was already applied in Australia [3, 15], where risk factors and breeding practices were evaluated before allowing herds to enter a certification program. Of all control methods, changing herd management is the most important factor affecting the efficacy of paratuberculosis control [5]. Calf-hygiene strategies have been found to be critically important in the USA paratuberculosis control program and most are economically attractive for midsize USA MAP-infected dairy farms [9].

\subsection{Certification schemes in other countries}

Certification schemes have been developed in several other countries, such as the USA, Australia, and more recently France $[3,8]$. In most of the schemes, yearly herd testing is performed. Seropositive animals are generally confirmed by fecal culture, which decreases the risk of a false positive result because fecal culture is almost $100 \%$ specific (especially at the herd level). If such an additional test on test positive animals is not performed, the certified status will not be sustainable.

In the Dutch program, all cattle older than 24 months are tested. Compared to Dutch dairy cattle herds, USA and Australian dairy herds are generally larger, with 100 or more cows plus additional young stock. In practice, testing all the animals of such a large herd with less than $100 \%$ specific test could lead to false positive results. In the model, a decrease in sampling proportion from 1.0 to 0.3 only marginally increases the number of $S_{1}$ herds after 25 years, whereas the risk that non-certified herds buy infected cattle from group 1 may increase. However, for herds with a given prevalence $p$, more animals are infected in larger rather than in smaller herds, increasing the probability of detecting the herd. Moreover, there is a balance between the time to detect infected herds and the time to clear them by chance. An interesting alternative for large herds that could be further investigated is to test only a sample of the animals to identify the herd health status but to restrict purchases to the negative-tested animals only.

Certification-and-monitoring programs can become useless if tests do not have a perfect specificity, especially when the withinherd prevalence decreases and false positive results increase. In programs that use a test with a perfect specificity or that reduce the risk of false positive results by confirming positive results with a second test that is $100 \%$ specific, certified herds keep their status over time and undetected but infected herds become detected or cleared. However, test-and-cull strategies alone cannot lead to a rapid increase in certified and truly MAP-free herds. On the one hand, a restriction of purchases to animals from herds at the highest level of certification and, on the other hand, a decrease in the within-herd prevalence, as can be obtained by improving herd management, may increase the number of certified truly MAP-free herds in a 25-year horizon.

\section{ACKNOWLEDGMENTS}

The authors thank A.A. de Koeijer and H.J.W. van Roermund for initial discussions and two anonymous referees for their detailed comments that greatly improved the paper.

\section{REFERENCES}

[1] Benedictus G., Dijkhuizen A.A., Stelwagen J., Economic losses due to paratuberculosis in dairy cattle, Vet. Rec. 121 (1987) 142-146.

[2] Bolske G., Englund S., Ongoing research on paratuberculosis in Sweden, Acta Vet. Scand. 44 (2003) 275-278. 
[3] Bonnet J.-N., Gounot G., Guerin D., Petit H., Vindel E., Vialard J., Les enjeux de la certification, Rapport du groupe de travail relatif à la certification en paratuberculose bovine, ACERSA, France, 2002, 88 p.

[4] Christensen J., Gardner I.A., Herd-level interpretation of test results for epidemiologic studies of animal diseases, Prev. Vet. Med. 45 (2000) 83-106.

[5] Collins M.T., Paratuberculosis: review of present knowledge, Acta Vet. Scand. 44 (2003) 217-221

[6] Daniels M.J., Henderson D., Greig A., Stevenson K., Sharp J.M., Hutchings M.R., The potential role of wild rabbits Oryctolagus cuniculus in the epidemiology of paratuberculosis in domestic ruminants, Epidemiol. Infect. 130 (2003) 553-559.

[7] Daniels M.J., Hutchings M.R., Greig A., The risk of disease transmission to livestock posed by contamination of farm stored feed by wildlife excreta, Epidemiol. Infect. 130 (2003) 561-568.

[8] Dufour B., Pouillot R., Durand B., A cost/benefit study of paratuberculosis certification in French cattle herds, Vet. Res. 35 (2004) 69-81.

[9] Groenendaal H., Galligan D.T., Economic consequences of control programs for paratuberculosis in midsize dairy farms in the United States, J. Am. Vet. Med. Assoc. 223 (2003) 1757-1768.

[10] Groenendaal H., Nielen M., Jalvingh A.W., Horst S.H., Galligan D., Hesselink J.W.. A simulation of Johne's disease control, Prev. Vet. Med. 54 (2002) 225-245.

[11] Johnson-Ifearulundu Y., Kaneene J.B., Lloyd J.W., Herd-level economic analysis of the impact of paratuberculosis on dairy herds, J. Am. Vet. Med. Assoc. 214 (1999) 822-825.
[12] Jørgensen J.B., Survival of Mycobacterium paratuberculosis in slurry, Nord. Vet. Med. 29 (1997) 267-270.

[13] Kalis C.H.J., Hesselink J.W., Barkema H.W., Collins M.T., Culture of strategically pooled bovine faecal samples as a method to screen herds for paratuberculosis, J. Vet. Diagn. Invest. 12 (2000) 547-551.

[14] Kalis C.H.J., Collins M.T., Barkema H.W., Hesselink J.W., Certification of herds as free of Mycobacterium paratuberculosis infection: actual pooled faecal results versus certification model predictions, Prev. Vet. Med. 65 (2004) 189-204.

[15] Kennedy D.J., Allworth M.B., Progress in national control and assurance programs for bovine Johne's disease in Australia, Vet. Microbiol. 77 (2000) 443-451.

[16] Ott S.L., Wells S.J., Wagner B.A., Herd-level economic losses associated with Johne's disease on US dairy operations, Prev. Vet. Med. 40 (1999) 179-192.

[17] Sternberg S., Viske D., Control strategies for paratuberculosis in Sweden, Acta Vet. Scand. 44 (2003) 247-249.

[18] Sweeney R.W., Transmission of paratuberculosis, Vet. Clin. North Am. Food Anim. Pract. 12 (1996) 305-313.

[19] Weber M.F., Groenendaal H., van Roermund H.J.W., Nielen M., Simulation of alternatives for the Dutch Johne's disease certificationand-monitoring program, Prev. Vet. Med. 62 (2004) $1-17$.

[20] Whitlock R.H., Wells S.J., Sweeney R.W., van Tiem J., Elisa and fecal culture for paratuberculosis (Johne's disease): sensitivity and specificity of each method, Vet. Microbiol. 77 (2000) 387-398. 